$11,15,16,19$

\title{
Исследование активационного барьера кристаллизации метастабильной жидкости методом метадинамики
}

\author{
(C) Е.О. Розанов ${ }^{1,2}$, С.П. Проценко ${ }^{1}$, В.Г. Байдаков ${ }^{1}$ \\ ${ }^{1}$ Институт теплофиизики УрО РАН, \\ Екатеринбург, Россия \\ 2 Уральский фредеральный университет, \\ Екатеринбург, Россия \\ E-mail: vvecme10@gmail.com
}

Поступила в Редакцию 8 июля 2021 г.

В окончательной редакции 13 июля 2021 г.

Принята к публикации 16 июля 2021 г.

При молекулярно-динамическом моделировании методом метадинамики исследована кристаллизация слабо переохлажденной леннард-джонсовской жидкости. Для описания фазового превращения в качестве коллективных переменных метадинамики выбраны параметр порядка Стейнхардта $Q_{6}$ группы частиц вокруг случайно выбранного атома и потенциальная энергия системы. Прослежено изменение коллективных переменных при преодолении активационного барьера кристаллизации. Получены данные о работе образования критического зародыша.

Ключевые слова: метадинамика, леннард-джонсовская жидкость, метастабильность, активационный барьер.

DOI: $10.21883 /$ FTT.2021.11.51582.04s

\section{1. Введение}

Жидкость за линией фазового равновесия жидкостькристалл может конечное время существовать в метастабильном (переохлажденном) состоянии. Метастабильное фазовое состояние - это состояние частичного равновесия термодинамической системы, которому соответствует локальный минимум термодинамического потенциала $G$. При заданных условиях абсолютно устойчивому, стабильному состоянию отвечает глобальный минимум термодинамического потенциала. Переохлажденная жидкость кристаллизуется после спонтанного образования жизнеспособного критического зародыша, с появлением которого преодолевается энергетический барьер, разделяющий метастабильное и стабильное состояния. Высота активационного энергетического барьера $\Delta G$, равная работе образования критического зародыша $W^{*}$, и размер зародыша $R^{*}$ зависят от глубины проникновения за линию фазового равновесия. С углублением в область метастабильных состояний $\Delta G$ и $R^{*}$ уменьшаются, а вероятность спонтанного образования зародышей растет.

Высоту активационного энергетического барьера $\Delta G=W^{*}$ можно рассчитать через частоту зародышеобразования $J$. Эти величины в классической теории нуклеации (КТН) связаны как

$$
J=\rho_{l} B \exp \left(-\Delta G / k_{\mathrm{B}} T\right),
$$

где $\rho_{l}-$ плотность жидкости, $B-$ кинетический множитель, $k_{\mathrm{B}}$ - постоянная Больцмана, $T$ - температура. Частота зародышеобразования может быть получена в натурных экспериментах и рассчитана при молекулярнодинамическом (МД) моделировании. Большинство экспериментальных исследований кинетики зародышеобразования в переохлажденной жидкости проведено при давлениях, близких к атмосферному, т. е. в области слабой метастабильности. Напротив, исследование фазовых превращений из состояний, близких к линии равновесия фаз, при прямом МД-моделировании затруднено. Вследствие малой вероятности спонтанного появления критического зародыша время ожидания фазового перехода в микроскопической системе на многие порядки превышает доступное время моделирования.

В настоящей работе для исследования активационного барьера кристаллизации при МД-моделировании был применен метод метадинамики [1]. С помощью этого метода можно ускорить выход системы из метастабильного состояния и напрямую получить информацию о высоте барьера при низких и умеренных степенях метастабильности.

\section{2. Метадинамическое моделирование}

В методе метадинамики система взаимодействующих частиц в процессе МД-моделирования выводится из метастабильного состояния путем добавления к ее гамильтониану потенциала смещения. Этот потенциал $V\left[\mathbf{s}\left(\mathbf{r}^{N}, t\right)\right]$ зависит от времени и коллективных переменных - функций положений $N$ частиц системы, и задается как ряд гауссовских функций, вносимых с шагом $\tau$ по времени. Такое преобразование гамильтониана меняет динамику системы и заставляет ее быстрее переходить в неисследованные состояния и реже возвращаться к 


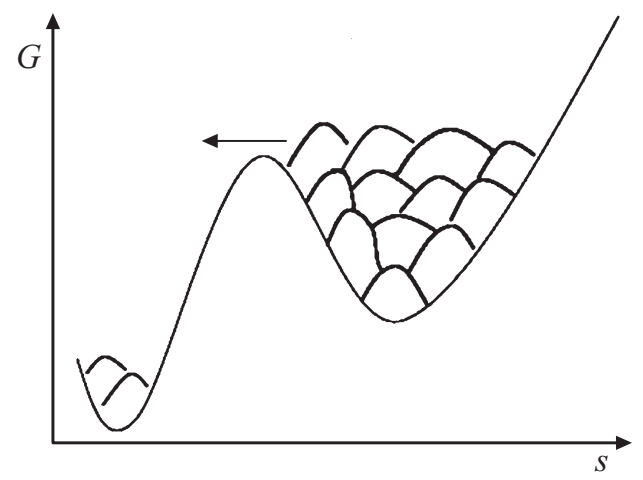

Рис. 1. Пример ландшафта термодинамического потенциала для одномерной системы. $G$ - термодинамический потенциал системы, $s$ - коллективная переменная, описывающая систему. Правая яма отвечает метастабильному состоянию, левая стабильному состоянию, дуги - добавляемые гауссианы.

уже посещенным. После заполнения потенциальной ямы гауссианами система переходит в стабильное состояние. На рис. 1 приведен пример заполнения метастабильной потенциальной ямы одномерной системы.

В конце моделирования ландшафт свободной энергии системы можно восстановить, построив все добавленные гауссианы в поле коллективных переменных. При достаточно длительном времени моделирования термодинамический потенциал системы есть

$$
G(\mathbf{s})=-\lim _{t \rightarrow \infty} V\left[\mathbf{s}\left(\mathbf{r}^{N}, t\right)\right] .
$$

Чтобы эффективно использовать метод МД-моделирования и правильно восстановить ландшафт термодинамического потенциала, необходимо заполнить гауссианами все потенциальные ямы возможных состояний системы. Однако для оценки высоты активационного барьера в случае кристаллизации достаточно дождаться его преодоления в ходе моделирования и кристаллизации всей системы [2].

\section{3. Параметры моделирования}

В работе исследовалась кристаллизация переохлажденной леннард-джонсовской (ЛД) жидкости. Система из 5324 ЛД-частиц размещалась в кубической ячейке с периодическими граничными условиями. Межчастичные взаимодействия рассчитывались на расстоянии $6.78 \sigma$ $(\sigma$ - расстояние, на котором межчастичный потенциал равен нулю). МД-моделирование проводилось при постоянных давлениях $p=-0.995,0$ и 6.912 (в единицах $\varepsilon / \sigma^{3}$, где $\varepsilon-$ энергетический параметр взаимодействия потенциала ЛД) и температурах $T=0.55$ и 0.7 (в единицах $k_{\mathrm{B}} / \varepsilon$ ) при различных степенях метастабильности. При каждом заданном значении давления переохлаждение варьировалось изменением температуры. При заданной температуре различные степени пересыщения достигались за счет изменения плотности (дав-

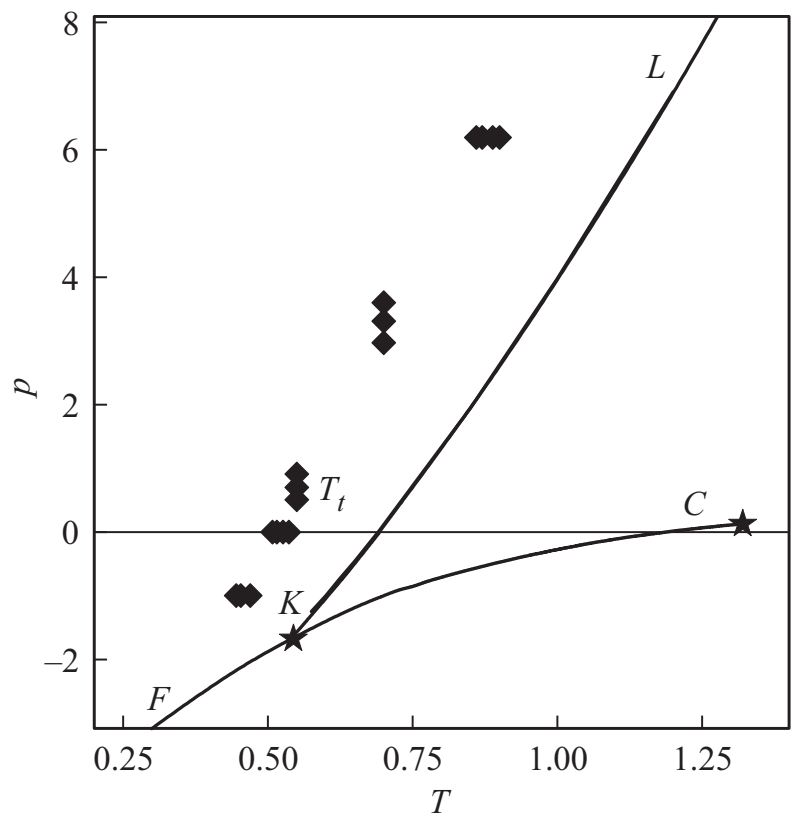

Рис. 2. Фазовая диаграмма ЛД системы в координатах $p-T$. Ромбики - исследованные состояния, $K L-$ линия плавления [3], $F C$ - спинодаль растянутой жидкости, $C-$ критическая точка, $T_{t}-$ тройная точка, $K-$ конечная точка линии плавления.

ления). На рис. 2 показаны исследованные термодинамические состояния. В каждом из них регистрировалось до 40 независимых событий кристаллизации.

В качестве коллективных переменных выбраны параметр порядка Стейнхардта $Q_{6}$ [4] для группы из 350 атомов вокруг случайно выбранной частицы и потенциальная энергия системы $U$. Ширина гауссианов равнялась 0.01 и 40 для параметра $Q_{6}$ и потенциальной энергии соответственно, высота гауссианов $1 k_{\mathrm{B}} T$. МДмоделирование проводилось в среде LAMMPS [5] с использованием пакета PLUMED v2.6.0 [6] для реализации метадинамического процесса.

\section{4. Результаты моделирования}

В результате моделирования были получены ландшафты термодинамического потенциала ЛД-системы. Выход жидкой фазы из состояния переохлаждения связан вначале с совершенствованием порядка в ограниченном объеме (увеличение $Q_{6}$ при слабом изменении $U$ ), а после достижения переходного состояния (образования критического зародыша) - с кристаллизацией всей системы (уменьшение $U$ ). На рис. 3 показан ландшафт термодинамического потенциала системы, где видно изменение коллективных переменных. Высоту барьера кристаллизации, равную работе образования критического зародыша, можно найти, измерив глубину потенциальной ямы метастабильного состояния относительно уровня переходного состояния на ландшафте термодинамического потенциала. 

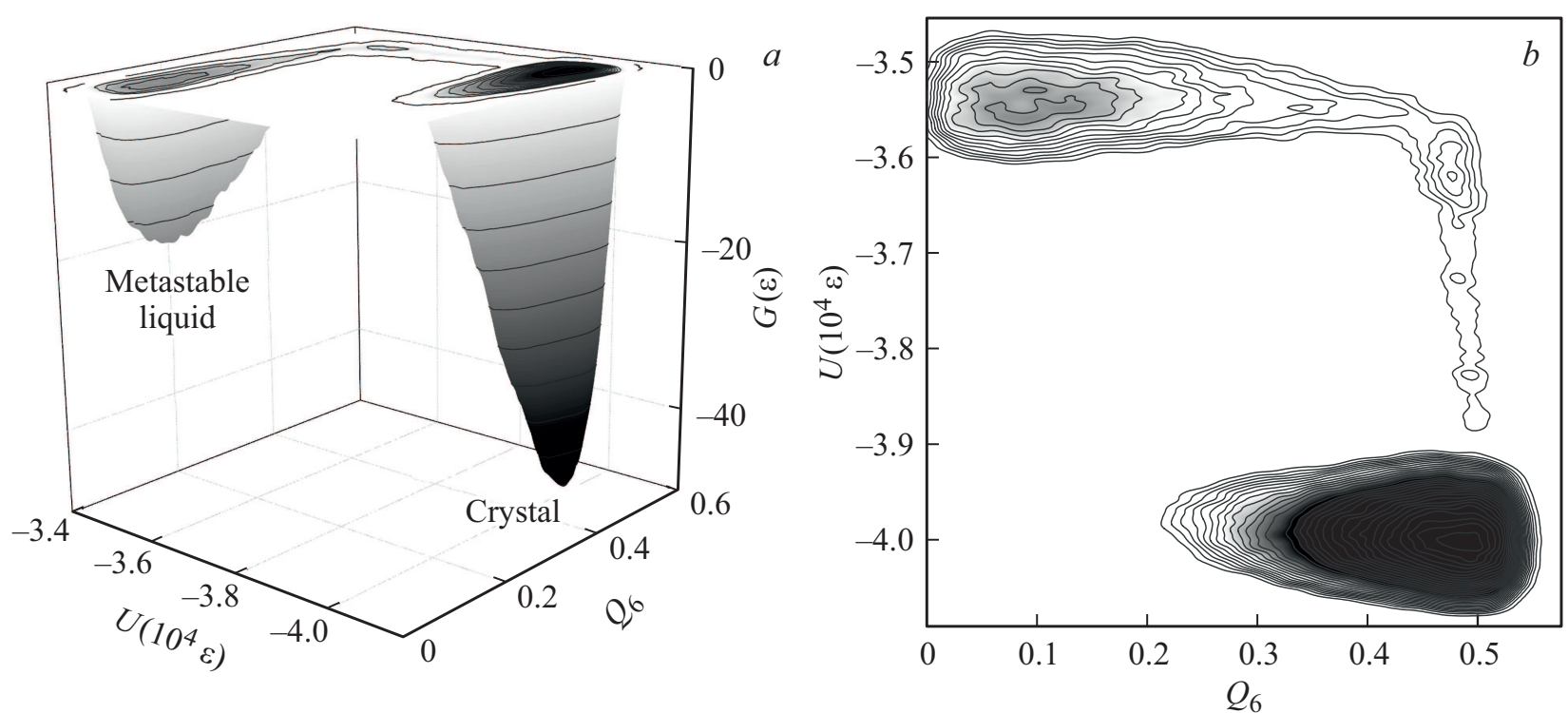

Рис. 3. a) Ландшафт свободной энергии для состояния $p=-0.995, T=0.469 . b)$ Проекция того же ландшафта в координа$\operatorname{Tax} Q_{6}-U$.

На рис. 4 показаны рассчитанные с помощью метадинамики значения приведенной работы образования критического зародыша $W^{*} / T$ при постоянных давлениях. Эти данные сопоставляются с результатами расчетов $W^{*} / T$ Байдакова и Проценко [7], вычисленными по КТН (ур. (1)) и МД-данным о частоте кристаллизации. Результаты двух подходов хорошо согласуются.

На рис. 5 показаны величины $W^{*} / T$ для изотерм, рассчитанные методом метадинамики, а также $W^{*} / T$,

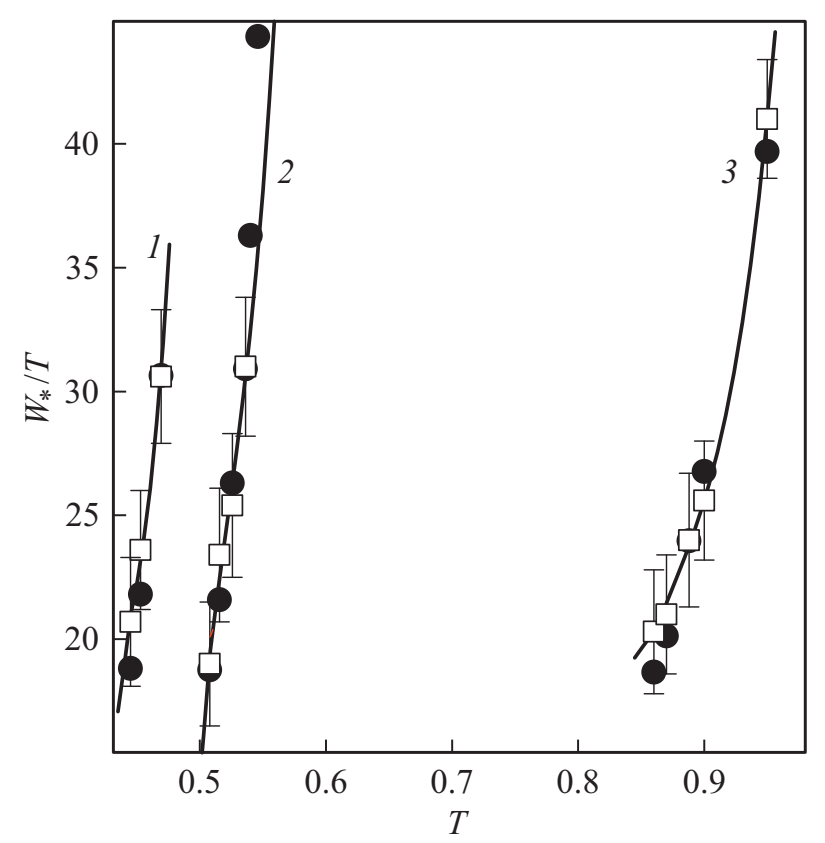

Рис. 4. Зависимость приведенной работы образования критического зародыша от температуры при давлениях $p=-0.995$ (кривая 1$), p=0$ (2) и $p=6.912(3)$. Квадраты - метод метадинамики, круги - расчет по данным о частоте нуклеации [7].

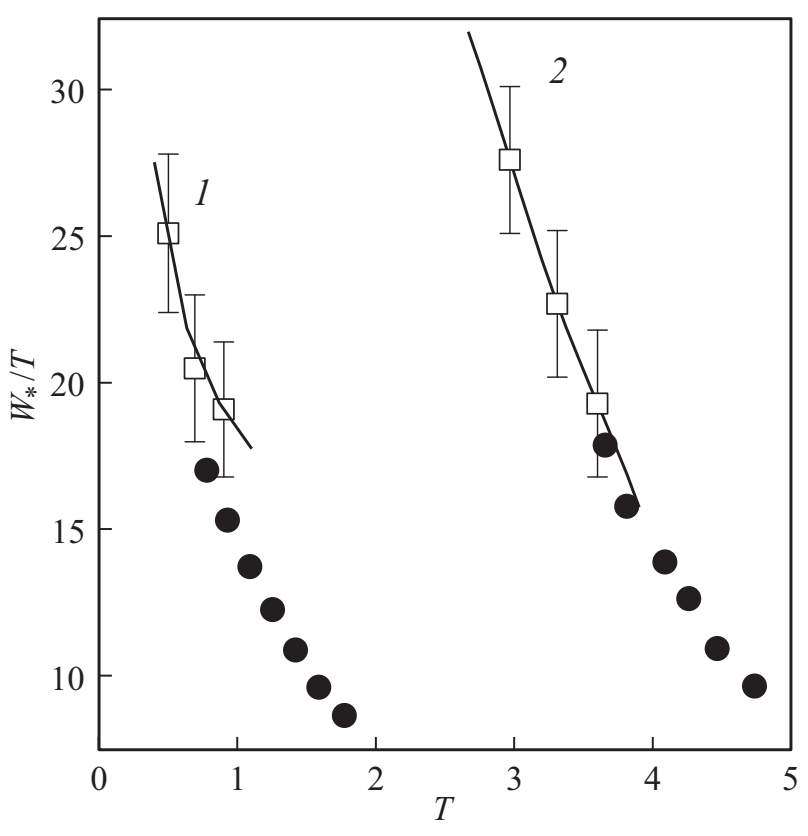

Рис. 5. Зависимость приведенной работы образования критического зародыша от давления при температурах $T=0.55$ (1) и $T=0.7$ (2). Квадраты - метод метадинамики, круги расчет по данным о частоте нуклеации [7].

полученные по данным о частоте зародышеобразования [7]. При постоянных температурах МД-моделирование кристаллизации проведено в метастабильных расстояниях, расположенных ближе к линии плавления, чем МД-расчеты в работе [7]. В случае температуры $T=0.7$ значения приведенной работы образования критического зародыша лежат на продолжении зависимости $W^{*} / T(p)$, полученной в [7]. При $T=0.55$ результаты МД-расчетов 
лежат выше зависимости $W^{*} / T(p)$ работы [7]. Рассогласование может быть вызвано тем, что выбор группы из 350 частиц для расчета коллективной переменной $Q_{6}$ в метадинамическом процессе далек от размера критического зародыша при данной температуре.

\section{5. Заключение}

В результате работы была доказана применимость метода метадинамики для исследования активационного барьера кристаллизации. Найденные высоты барьера для изобар согласуются с данными, полученными прямым МД-расчетом частоты кристаллизации. С помощью метода метадинамики были исследованы области метастабильности, в которых невозможен расчет работы образования критического зародыша при классическом МД-моделировании. Показано, что для корректного расчета активационного барьера необходима калибровка использованных коллективных переменных, описывающих систему.

В дальнейшем будет исследована возможность МД-моделирования кристаллизации с другими коллективными переменными, такими как $Q_{6}$ для большего числа атомов и число атомов в наибольшем зародыше в системе. Также будет исследована применимость метадинамики при моделировании таких фазовых переходов, как кавитация кристалла и жидкости.

\section{Благодарности}

Авторы благодарят Институт математики и механики имени Н.Н. Красовского УрО РАН за предоставление ресурсов суперкомпьютера „Уран“ для проведения расчетов.

\section{Финансирование работы}

Работа выполнена при поддержке проекта РФФИ 2048-660027 p_a.

\section{Конфликт интересов}

Авторы заявляют, что у них нет конфликта интересов.

\section{Список литературы}

[1] A. Laio, M. Parrinello. Proceed. Natl Acad. USA 99, 20, 12562 (2002).

[2] P. Raiteri, A. Laio, F.L. Gervasio, C. Micheletti, M. Parrinello. J. Phys. Chem. B 110, 8, 3533 (2006).

[3] V.G. Baidakov, S.P. Protsenko. Phys. Rev. Lett. 95, 1, 015701 (2005).

[4] P.J. Steinhardt, D.R. Nelson, M. Ronchetti. Phys. Rev. B 28, 2, 784 (1983).

[5] S. Plimpton. J. Comp. Phys. 117, 1, 1 (1995). LAMMPS Molecular Dynamics Simulator. URL: https://lammps.sandia. gov/ (Accessed: 03.02.2020).

[6] The PLUMED Consortium. Nature Methods 16, 8, 670 (2019). G.A. Tribello, M. Bonomi, D. Branduardi, C. Camilloni, G. Bussi. Comp. Phys. Comm. 185, 2, 604 (2014).

[7] V.G. Baidakov, K.R. Protsenko. J. Phys. Chem. B 123, 38, 8103 (2019).

Редактор Е.В. Толстякова

Продолжение публикации материалов семинара в ФТТ № 12/21 